\title{
Mission Reliability Modeling Based on Logic Diagram
}

\author{
Jiaxin JIANG \& Xiaohuan ZHU \\ Southwest Research Institute of Electronic Equipment, Chengdu, China
}

Yaping LI

Shanghai Institute of System Engineering, Shanghai, China

\begin{abstract}
Based on the analysis of reliability block diagram and reliability model, the error of mission reliability bridge-model can be inferred. The error cause was worked out by the enumerations data set and the total probability formula, which proved that the inference is right, while the bridge-model of mission reliability was found correctly. A new modeling method, which based on mission profile logic diagram, was put forward with comparison of relation between logic gate and mission reliability model, to solve modeling difficulty for complex system, which need high level logical thinking while error is yet easily made. Application result shows that the method can express product mission profile logically, and get exact reliability model easily. According to comparison of relation between reliability logic diagram and fault tree, an indirect method was presented for complex system which was to get mission reliability through calculating top event probability of FT.
\end{abstract}

KEYWORD: Mission Reliability; Modeling of Reliability; Fault Tree; Logic Diagram

Mission Reliability [1-3] is an important indicator to measure the ability to work in military equipment. With the development and production of military equipment, mission reliability index become more prominent.

Currently, research in the field of reliability, the common mission reliability modeling methods include [4-9]: Monte Carlo simulation analysis, based on probability theory and mathematical analytical method Markov chain, based on reliability diagrams and fault tree graphics deductive.

Among them, the Monte Carlo[8-12] simulation analysis method for assessing the reliability of large, complex systems have a good assessment of the effect, especially for different failure distribution between devices in the same system case analysis can be programmed with the calculation accuracy Features.

Based on probability theory and mathematical analysis of Markov chains[13-14], that is mainly applied to constant failure rate and repairable system. With the introduction of fuzzy theory and cloud theory Markov reliability model, to a certain extent, solve the problem of vagueness and randomness.

For complex systems, these two methods have its obvious advantages, the Monte Carlo method is suitable for computer simulation analysis, and the Markov chain is suitable for mathematical modeling and analysis. But for the engineering practice, the biggest problem is the existence of the analysis process is too professional, so the reliability engineer must to have strong professional background requirements; and logical analysis process is not clear, hard to audit.

Currently, the most widely used is still the reliability diagram analysis and probabilistic modeling method of combining [1-3]. Establish the reliability diagram analysis according to the mission profile, is one of the main methods to carry out reliability modeling assessment mission reliability. For simple system which can be evaluated by conventional mission reliability model, the method is relatively fast and accurate. But for complex systems, due to its implementation logic intertwined, extremely complex, requiring personnel strong logical thinking to complete, otherwise error-prone.

In this paper, it is carried out that mission reliability modeling based on logic diagram. Not only clear logic analysis process, but also conducive to the audit, avoid modeling errors. This application has a strong role in guiding the project.

\section{EXAMPLE ANALYSIS}

\subsection{Application Error}

Fig 1 is the bridge-model. In the "Model Reliability Engineering Handbook", the mathematic model is 


$$
\begin{aligned}
R_{S}(t)= & R_{1}(t) R_{3}(t)+R_{2}(t) R_{4}(t)+R_{1}(t) R_{Q}(t) R_{4}(t) \\
& +R_{2}(t) R_{Q}(t) R_{3}(t)-R_{1}(t) R_{2}(t) R_{3}(t) R_{4}(t) \\
& -R_{1}(t) R_{2}(t) R_{4}(t) R_{Q}(t)-R_{1}(t) R_{2}(t) R_{3}(t) R_{Q}(t) \\
& +2 R_{1}(t) R_{2}(t) R_{3}(t) R_{4}(t) R_{Q}(t)
\end{aligned}
$$

Where $R_{s}(t)=$ reliability of system;

$R_{1}(t)=$ reliability of component 1 ;

$R_{2}(t)=$ reliability of component 2 ;

$R_{3}(t)=$ reliability of component 3 ;

$R_{4}(t)=$ reliability of component 4 ;

$R_{Q}(t)=$ reliability of component $Q$;

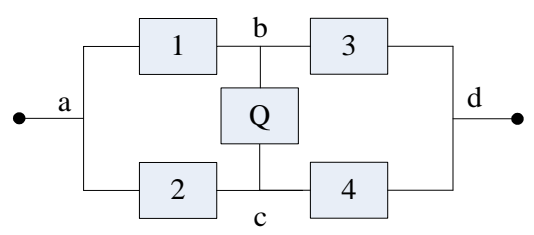

Figure 1. Bridge-model

Through the observation can be seen in Figure 1, the combination 1 (component 1 and 3) and the combination 2 (component 2 and 4) can be collocated swap, the combination 3 (component 1 and 2) and the combination 4 (component 3 and 4) can also be collocated swap, so it can be inferred that, in its reliability model mathematical expressions, should satisfy the following characters:

Character1: $\quad R_{1}(t) R_{3}(t)$ and $R_{2}(t) R_{4}(t)$ can be collocated swap;

Character2: $\quad R_{1}(t) R_{2}(t)$ and $R_{3}(t) R_{4}(t)$ can be collocated swap.

Through the observation of Equation (1) can be found that, if the existence of monomial $-R_{1}(t) R_{2}(t) R_{4}(t) R_{Q}(t)$ is correct, Then,

According to the character 1 can be launched, there should be a monomial $-R_{1}(t) R_{2}(t) R_{3}(t) R_{Q}(t)$

Conclusion1.

According to the character 2, combination 3 and 4 can be collocated swap; there should be a monomial $-R_{2}(t) R_{3}(t) R_{4}(t) R_{Q}(t)$

Conclusion2.

Similarly, according to the conclusion 1 , there should be a monomial $-R_{1}(t) R_{3}(t) R_{4}(t) R_{Q}(t)$

Conclusion3.

The Equation (1) only satisfies Character1 (Conclusion 1), does not satisfy Character 2 (Conclusion 2 and 3). Therefore it can be concluded that this mission reliability model is error.

\subsection{Total probability formula}

Through the analysis, component $Q$ as the key component in the reliability diagram, respectively, no fault and fault of two mutually exclusive states. According to the total probability theorem, this reliability diagram can be divided into two states for analysis.

State 1: component $Q$ is not fault. Then the reliability diagram can be converted to figure 2 .

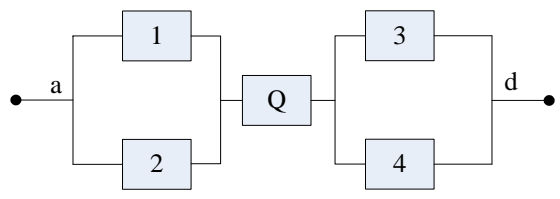

Figure 2. Bridge model in State 1

The mathematical model of reliability is

$$
\begin{aligned}
R_{S 1}(t)= & R_{Q}(t)\left[1-\left(1-R_{1}(t)\right)\left(1-R_{2}(t)\right)\right] \\
& \times\left[1-\left(1-R_{3}(t)\right)\left(1-R_{4}(t)\right)\right]
\end{aligned}
$$

State 2: component $Q$ is fault. Then the reliability diagram can be converted to figure 3 .

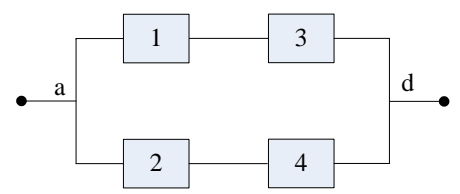

Figure 3. Bridge model in State 2

The mathematical model of reliability is

$$
\begin{aligned}
R_{S 2}(t)= & \left(1-R_{Q}(t)\right)\left[1-\left(1-R_{1}(t) R_{2}(t)\right)\right] \\
& \times\left[1-\left(1-R_{3}(t) R_{4}(t)\right)\right]
\end{aligned}
$$

State 1 and 2 is mutually exclusive events. According to Bayes' theorem, system reliability is the sum of two states. So

$$
R_{S}(t)=R_{S 1}(t)+R_{S 2}(t)
$$

Solving with Equation (1) and (2), then

$$
\begin{aligned}
R_{S}(t)= & R_{1}(t) R_{3}(t)+R_{2}(t) R_{4}(t)+R_{1}(t) R_{Q}(t) R_{4}(t) \\
& +R_{2}(t) R_{Q}(t) R_{3}(t)-R_{1}(t) R_{2}(t) R_{3}(t) R_{4}(t) \\
& -R_{1}(t) R_{2}(t) R_{4}(t) R_{Q}(t)-R_{1}(t) R_{2}(t) R_{3}(t) R_{Q}(t) \\
& -R_{1}(t) R_{3}(t) R_{4}(t) R_{Q}(t)-R_{2}(t) R_{3}(t) R_{3}(t) R_{Q}(t) \\
& +2 R_{1}(t) R_{2}(t) R_{3}(t) R_{4}(t) R_{Q}(t)
\end{aligned}
$$

Where $R_{s}(t)=$ reliability of system;

$R_{1}(t)=$ reliability of component 1 ;

$R_{2}(t)=$ reliability of component 2 ;

$R_{3}(t)=$ reliability of component 3 ;

$R_{4}(t)=$ reliability of component 4 ;

$R_{Q}(t)=$ reliability of component $Q$;

Equation (5) is different with (1), and satisfies the Character 1) and 2), while satisfying the Conclusion 1,2 and 3.

Combining Equation (1) and (5), it can be seen 
1) The set $\left\{X_{1} X_{3}\right\}$ and $\left\{X_{1} X_{4} X_{Q}\right\}$ contain elements $X_{1} \bar{X}_{2} X_{3} X_{4}$

2) The set $\left\{X_{2} X_{4}\right\}$ and $\left\{X_{2} X_{3} X_{Q}\right\}$ contain elements $\bar{X}_{1} X_{2} X_{3} X_{4}$.

$R_{1}(t) R_{3}(t) R_{4}(t) R_{Q}(t)$ and $R_{2}(t) R_{3}(t) R_{4}(t) R_{Q}(t)$ are counted twice, should be subtracted from Equation (1).

Therefore, Equation (1) is an error model; the correct bridge-model is Equation (5).

\subsection{Result analysis}

By example it can be seen that for complex systems, according to the mission profile model directly, not only for logical thinking person demanding, illconsidered and easy situation. Hence it need for effective ways to support the process of logical analysis, modeling guidance.

\section{BUILDING MISSION RELIABILITY LOGIC DIAGRAM}

\subsection{Relationship between logic gates and reliability models}

\subsubsection{Direct correspondence relationship}

Assuming the top event is a fault, the correspondence between the common reliability models and logic gates, as shown in Table 1.

Table 1. Relationship between logic gates and models

\begin{tabular}{|c|c|l|c|}
\hline \multicolumn{2}{|c|}{ Logic gate } & \multicolumn{1}{|c|}{ Indicate } & Model \\
\hline AND gate & $\begin{array}{l}\text { An output event occurs } \\
\text { only when all the input } \\
\text { events have occurred }\end{array}$ & $\begin{array}{c}\text { Serial } \\
\text { Model }\end{array}$ \\
\hline OR gate & $\begin{array}{l}\text { An output event occurs if } \\
\text { at least one of the input } \\
\text { events has occurred }\end{array}$ & $\begin{array}{c}\text { Parallel } \\
\text { Model }\end{array}$ \\
\hline Conditional \\
AND gate & - & $\begin{array}{l}\text { An output event occurs if } \\
\text { at least R of the N input } \\
\text { events have occurred }\end{array}$ & $\begin{array}{r}\text { R/N } \\
\text { Model }\end{array}$ \\
\hline
\end{tabular}

\subsubsection{Indirect correspondence relationship}

For complex mission reliability model, through functional logic diagram, it can be calculated. Thus, for the bridging model of Figure 1, the establishment of functional logic diagram in Figure 4.

By logical calculations, the minimum cut set is $\left\{X_{1}, X_{3}\right\},\left\{X_{2}, X_{4}\right\},\left\{X_{1}, X_{Q}, X_{4}\right\},\left\{X_{2}, X_{Q}, X_{3}\right\}$

Therefore, the top event $T$ can be expressed as

$$
T=E_{0}=X_{1} X_{3}+X_{2} X_{4}+X_{1} X_{Q} X_{4}+X_{2} X_{Q} X_{3}
$$

According to disjoint algorithm, let

$$
K_{1}=X_{1} X_{3}, K_{2}=X_{2} X_{4}, K_{3}=X_{1} X_{Q} X_{4}, K_{4}=X_{2} X_{Q} X_{3}
$$

Then $K_{1}+K_{2}+K_{3}+K_{4}$

$$
\begin{aligned}
= & K_{1}+\bar{K}_{1} K_{2}+\bar{K}_{1} \bar{K}_{2} K_{3}+\bar{K}_{1} \bar{K}_{2} \bar{K}_{3} K_{4} \\
= & X_{1} X_{3}+\left(\overline{X_{1} X_{3}}\right) X_{2} X_{4} \\
& +\left(\overline{X_{1} X_{3}}\right)\left(\overline{X_{2} X_{4}}\right) X_{1} X_{Q} X_{4} \\
& +\left(\overline{X_{1} X_{3}}\right)\left(\overline{X_{2} X_{4}}\right)\left(X_{1} X_{Q} X_{4}\right) X_{2} X_{Q} X_{3} \\
= & X_{1} X_{3}+\bar{X}_{1} X_{2} X_{4}+X_{1} \bar{X}_{3} X_{2} X_{4} \\
& +X_{1} \bar{X}_{2} \bar{X}_{3} X_{4} X_{Q}+\bar{X}_{1} X_{2} X_{3} \bar{X}_{4} X_{Q}
\end{aligned}
$$

Then, the probability $Q$ of the top event is

$$
\begin{aligned}
Q= & P\left(X_{1} X_{3}+\bar{X}_{1} X_{2} X_{4}+X_{1} \bar{X}_{3} X_{2} X_{4}\right. \\
& \left.+X_{1} \bar{X}_{2} \bar{X}_{3} X_{4} X_{Q}+\bar{X}_{1} X_{2} X_{3} \bar{X}_{4} X_{Q}\right) \\
= & R_{1} R_{3}+\left(1-R_{1}\right) R_{2} R_{4}+R_{1}\left(1-R_{3}\right) R_{2} R_{4} \\
& +R_{1}\left(1-R_{2}\right)\left(1-R_{3}\right) R_{4} R_{Q} \\
& +\left(1-R_{1}\right) R_{2} R_{3}\left(1-R_{4}\right) R_{Q}
\end{aligned}
$$

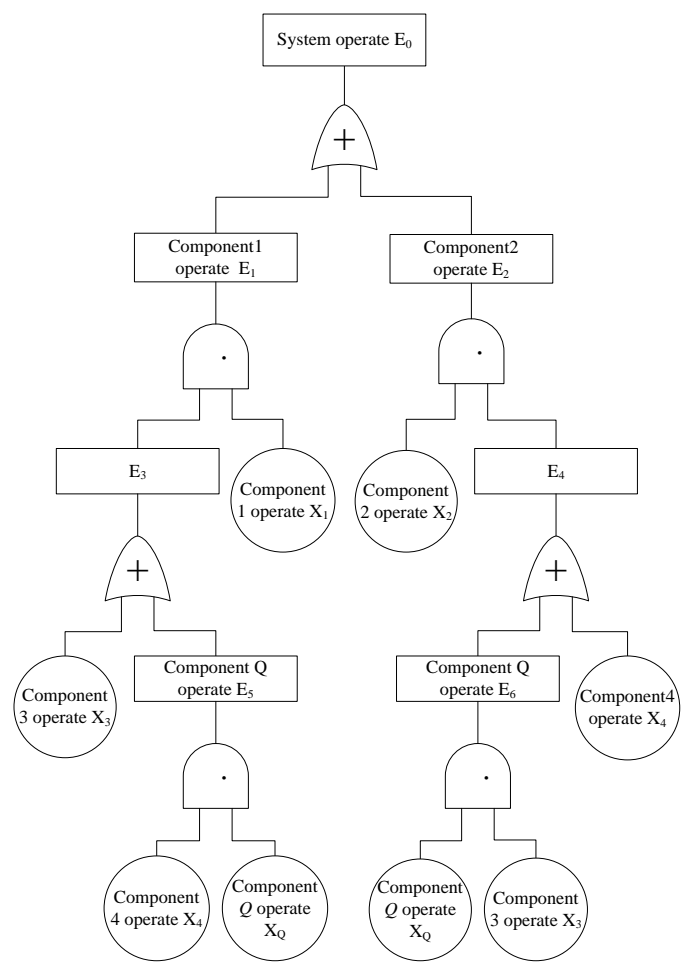

Figure 4. Bridge model in logic gate

Figure 4 is logic diagram of the bridge-model, so the probability $Q$ of the top event is the mission reliability $R_{s}(t)$ of the bridge-model. Solving

$$
\begin{aligned}
R_{S}(t)= & R_{1}(t) R_{3}(t)+R_{2}(t) R_{4}(t)+R_{1}(t) R_{Q}(t) R_{4}(t) \\
& +R_{2}(t) R_{Q}(t) R_{3}(t)-R_{1}(t) R_{2}(t) R_{3}(t) R_{4}(t) \\
& -R_{1}(t) R_{2}(t) R_{4}(t) R_{Q}(t)-R_{1}(t) R_{2}(t) R_{3}(t) R_{Q}(t) \\
& -R_{1}(t) R_{3}(t) R_{4}(t) R_{Q}(t)-R_{2}(t) R_{3}(t) R_{3}(t) R_{Q}(t) \\
& +2 R_{1}(t) R_{2}(t) R_{3}(t) R_{4}(t) R_{Q}(t)
\end{aligned}
$$

Where $R_{s}(t)=$ reliability of system;

$R_{1}(t)=$ reliability of component 1 ;

$R_{2}(t)=$ reliability of component 2 ; 
$R_{3}(t)=$ reliability of component 3 ;

$R_{4}(t)=$ reliability of component 4 ;

$R_{Q}(t)=$ reliability of component $Q$;

Equation (9) is the same with (5).

\subsection{Relationship between fault tree and mission reliability logic diagram}

Through the logic gate, the relationship between the fault tree and mission reliability logic diagram see Table 2

Table 2. Relationship between mission reliability logic diagram and fault tree

\begin{tabular}{|c|c|c|}
\hline & $\begin{array}{c}\text { Mission reliability } \\
\text { logic diagram }\end{array}$ & fault tree \\
\hline Define & $\begin{array}{l}\text { The probability that a } \\
\text { system (component) } \\
\text { will function over } \\
\text { some time period } t \text {. }\end{array}$ & $\begin{array}{l}\text { causal logic diagrams is } \\
\text { composed by event, logic } \\
\text { gate and transform sign, } \\
\text { likes a headstand tree. }\end{array}$ \\
\hline $\begin{array}{l}\text { Meaning of } \\
\text { Top event }\end{array}$ & System operate & System fault \\
\hline $\begin{array}{c}\text { Probability of } \\
\text { Top event }\end{array}$ & Mission reliability $R_{S}$ & Failure rate $F_{S}$ \\
\hline
\end{tabular}

According to compare, it can be seen that fault tree and mission reliability logic diagram are two sate of product, fault or operating. They are complementary relationship. Therefore the probability of failure can be solved through the fault tree, indirectly get mission reliability, while the system function logic is complicated, or in the mission reliability modeling.

\section{APPLICATION}

Figure 5 is a typical lighting circuit system. The physical structure is a parallel circuit composed of multiple identical lamps. In the absence of other fault premise, any lighting failure, shall not affect the other lighting.

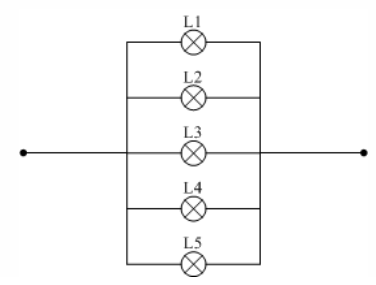

Figure 5. Physical structure of lighting system

This assumes that the "prescribed function" of this lighting system is defined in the following 5 cases, respectively.

\subsection{Case 1}

Case 1: All 5 lights work; any light fails, leading to failure of the system function. Then
Reliability logic diagram is shown in Figure 6; while the reliability block diagram is shown in Figure 7.
Figure 6. Logic diagram

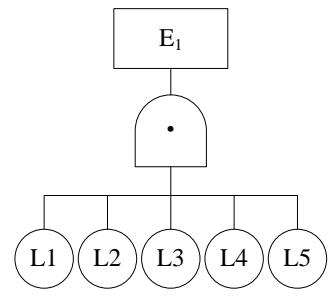

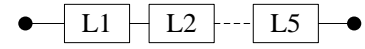

Figure 7. Block diagram
Mathematical model of mission reliability is the Equation (10)

$$
R_{s}(t)=\prod_{i=1}^{5} R_{i}(t)
$$

Where $R_{s}(t)=$ reliability of system; $R_{i}(t)=$ reliability of light $i$.

\subsection{Case 2}

Case 2: Any one light can work. Then

Reliability logic diagram is shown in Figure 8; while the reliability block diagram is shown in Figure 9.

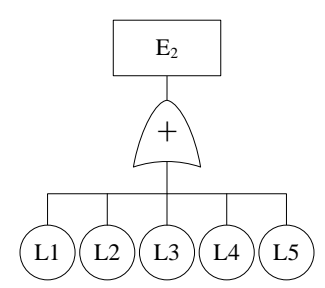

Figure 8. Logic diagram

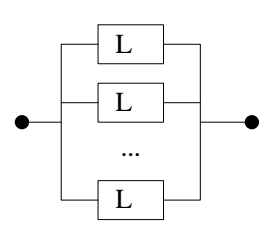

Figure 9. Block diagram
Mathematical model of mission reliability is the Equation (11)

$$
R_{s}(t)=1-\prod_{i=1}^{5}\left[1-R_{i}(t)\right]
$$

Where $R_{s}(t)=$ reliability of system;

$$
R_{i}(t)=\text { reliability of light } i \text {. }
$$

\subsection{Case 3}

Case 3: Any one light can work in conditional. Then

Reliability logic diagram is shown in Figure 10; while the reliability block diagram is shown in Figure 11.

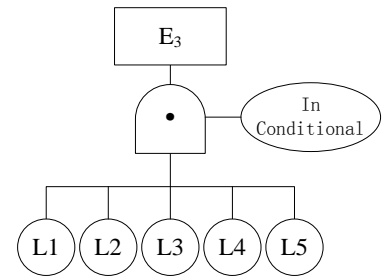

Figure 10. Logic diagram

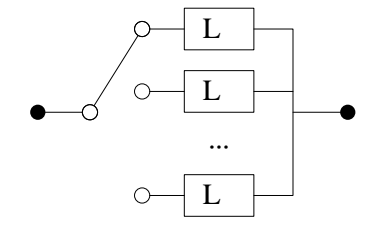

Figure 11. Block diagram 
Mathematical model of mission reliability is the Equation (12)

$$
R_{\mathrm{s}}(t)=R_{m} R_{i}\left(t-\sum_{j=1}^{i-1} t_{j}\right)
$$

Where $R_{s}(t)=$ reliability of system;

$R_{i}(t)=$ reliability of light $i$;

$t_{j}=$ operation time of light $i$ before failure;

$R_{m}=$ reliability of control switch.

\subsection{Case 4}

Case 4: Any 3 lights can work at least. Then

Reliability logic diagram is shown in Figure 12; while the reliability block diagram is shown in Figure 13.

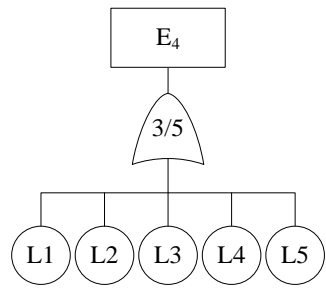

Figure 12. Logic diagram

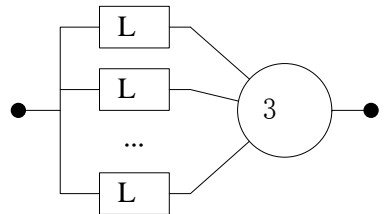

Figure 13. Block diagram
Mathematical model of mission reliability is the Equation (13)

$$
R_{\mathrm{s}}(t)=R_{m} \sum_{i=3}^{5}\left(C_{5}^{i} R(t)^{i}(1-R(t))^{5-i}\right)
$$

Where $R_{s}(t)=$ reliability of system;

$R(t)=$ reliability of light (every light is same);

$R_{m}=$ reliability of control switch.

\subsection{Case 5}

Case 5: At least one group work properly (two: L1 and 3 or L2 and 4) while L5 is failure; otherwise, at least one group work properly (two: L1 and 4 or L2 and 3) while L5 is operating. Then the reliability logic diagram is shown in Figure 4; while the reliability block diagram is shown in Figure 1.Mathematical model of mission reliability is the Equation (5) and (9).

\section{CONCLUSION}

Mission reliability modeling based on logic diagram, which can solve the following problem

1) Displayed intuitively logical relationship of the product function, effectively improve the accuracy of mission reliability modeling.

2) Achieving readability of product logic function; reducing the personnel requirement of logic thinking;
3) The calculation method of drawing fault tree top event can carry out the reliability modeling and computing by software, reducing the workload of manual modeling and failure rate.

4) Narrowing the gap between physical structure and reliability block diagram by logic diagram. Reliability staff may submit relevant comments and suggestions to improve the design by analyzing results, guide the reliable design.

\section{REFERENCES}

[1] Charles E. Ebeling. 2008. An Introduction to Reliability and Maintainability Engineering. Beijing: Tsinghua University Press.

[2] Gong Qingxiang, Zhao Yu, Gu Changhong. 2006. Project Reliability Engineering Handbook. Beijing: National Defense Industry Press.

[3] Kang Rui, Shi Rongde, Li Ruiying. 2010. Project Reliability Maintainability and Supportability Specification. Beijing: National Defense Industry Press.

[4] Sun Xiaozhe, Li Weiqi, Chen Zongji. 2011. Hierarchical Hybrid Reliability Modeling Method for Flight Control Computer System. Journal of Shanghai Jiaotong University 45(2): 277-283.

[5] Wang Yujue, Yang Jikun, Xu Tingxue, et al. 2013. Reliability Modeling and Evaluation for Complex System Based on Maximum Entropy. Ship Electronic Engineering 225(3): 80-99.

[6] Liu Yu. 2010. Multi-State Complex System Reliability Modeling and Maintenance Decision. Chengdu: University of Electronic Science and Technology of China,

[7] Liu Bin, Wu Xiaoyue. 2012. Mission Reliability Modeling of Missile Defense System Based on Phase Mission Bayesian Networks. Journal of Academy of Equipment Command \& Technology 23(1): 75-78.

[8] Zhang Yutao, Tang Jun, Zhang Mingqing, et al. 2008. Research on Reliability Simulation Process Model Based on Monte Carlo Method. Systems Engineering and Electronics30(7): 1374-1377

[9] Liu Qinwen, Zhao Pinlun. 2013. An Analysis Method of System Mission Reliability Based on Monte Carlo Method. Electronic Product Reliability and Environmental Testing31(5): 17-22

[10] Jin Xing, Hong Yanji. 2005. Monte Carlo Method of MTTF Evaluation for Large Complex System. Journal of System Simulation 17(1): 66-68.

[11] Fang Luebin, Cai Jinding. 2011. Reliability Assessment of Microgrid Using Sequential Monte Carlo Simulation. Journal of Electronic Science and Technology 9(1):31-34.

[12] Lu Zhong, Sun Youchao. 2008. Reliability Calculating Method of Complex Systems Based on Monte Carlo Method and Genetic Algorithm. Systems Engineering and Electronics 30(12): 2519-2522.

[13] Li Lirong. 2012. Method of Mission Reliability Analysis of Large PMS Based on Markov Model. Changsha: National University of Defense Technology.

[14] Yuan Xiukai, Lu Zhenzhou, Qiao Hongwei. 2010. Conditional Probability Markov Chain Simulation Based Reliability Analysis Method for Nonnormal Variables. Science China (Technological Sciences) 53(5):1434-1441. 